\title{
The Mayor's Speech
}

\section{George Dimopoulos}

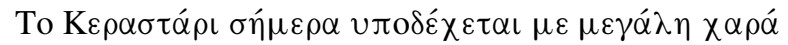

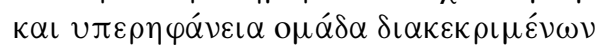

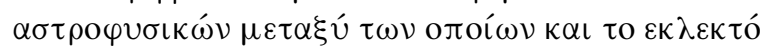

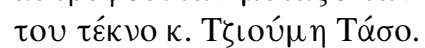

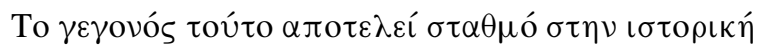

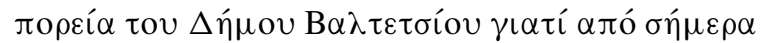

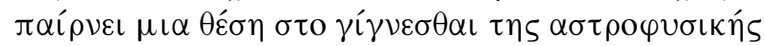

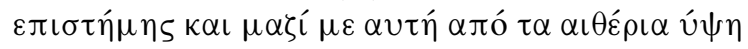

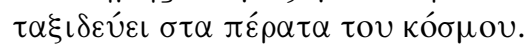

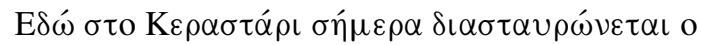

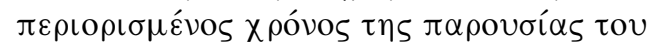

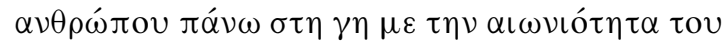

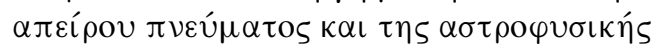

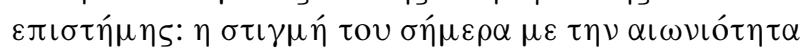

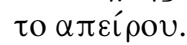

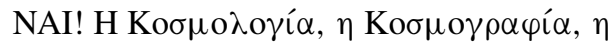

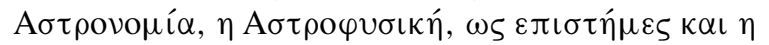

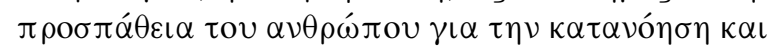

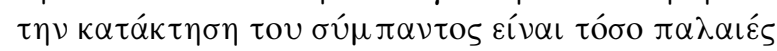

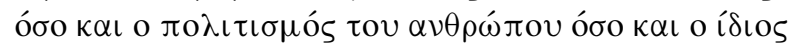

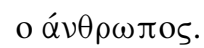

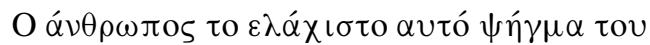

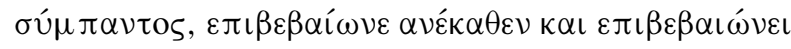

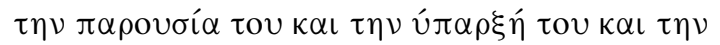

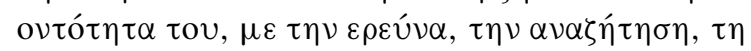

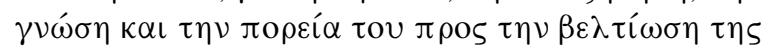
$\zeta \omega \eta \dot{s ~ \kappa \alpha l ~ \tau \eta \nu ~ \tau \varepsilon \lambda \varepsilon \varepsilon ́ \omega \sigma \eta ́ ~ \tau o v . ~}$

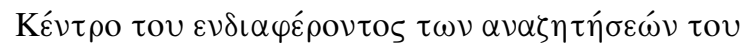

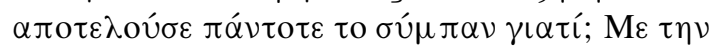

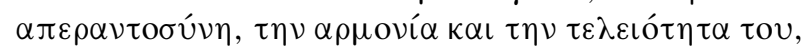

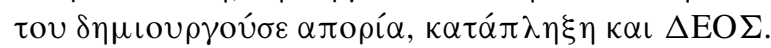

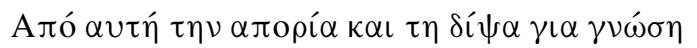

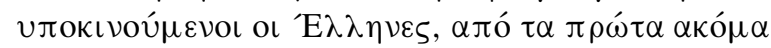

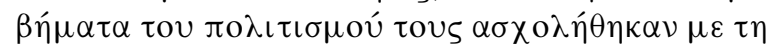

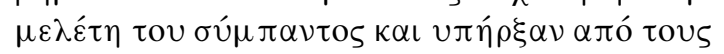

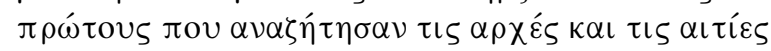

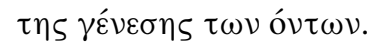

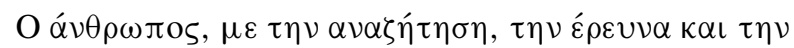

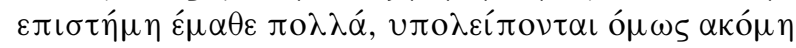

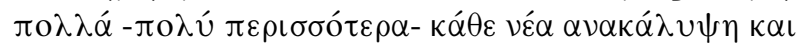

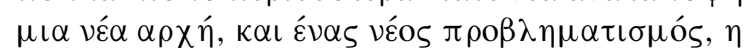

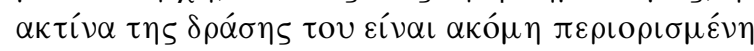

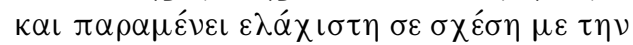

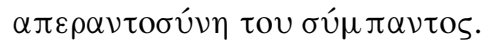

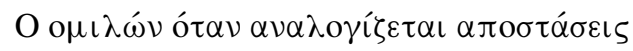

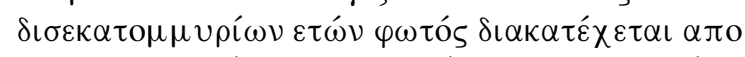

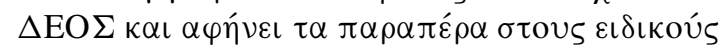

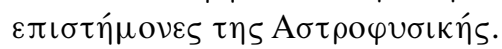

Today, Kerastari is welcoming, with great pleasure and pride, a group of distinguished astrophysicists, among which its exquisite child Mr Tassos Tzioumis.

Such an event is a stage in the history of Valtetsi district, since from now on this district partakes in the acts of astrophysics, whereas it travels and becomes known all around the world.

Today, in Kerastari, the limited existence of the human being on the earth meets the eternity of the immense spirit and astrophysics; today's time meets the eternity of infinity.

It's generally accepted that the science of cosmogony, cosmography, astronomy and astrophysics and the human's attempts to perceive and conquer the universe are as old as the human culture, or the human itself.

Human, who is a speck of the universe, has always confirmed his presence, existence, and individuality through research, wondering, knowledge and through his effort to improve his life and reach perfection.

The Universe has always been human's main interest. Why is this happening? It's the immensity, harmony and perfection of the universe itself that created his curiosity, astonishment and AWE.

Greeks, from the earliest steps of their culture, were instigated from this curiosity and eagerness for knowledge, which lead them to deal with the meditation of the universe. They were one of the first to inquire the principles and the causes of the beings' origin.

Human has learnt a great deal through quest, research and science; yet, there are a lot more to learn, since every discovery means a new Beginning and a new Consideration. His actions are still limited and remain minimum in accordance with the immensity of the Universe.

As the lecturer calls to mind the distance of the billion years of light he becomes possessed by the feeling of AWE and leaves the rest to the Astrophysics specialists. 


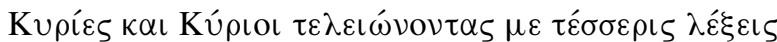
$\rho \eta \dot{\eta} \mu \alpha \tau \alpha \theta \alpha \pi \rho \circ \sigma \pi \alpha \theta \dot{\eta} \sigma \omega \nu \alpha \varepsilon \kappa \varphi \rho \alpha ́ \sigma \omega \tau \alpha$

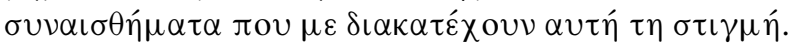

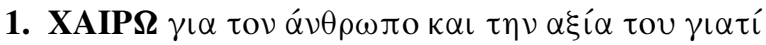

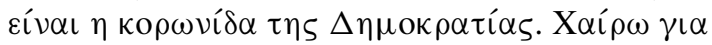
oas.

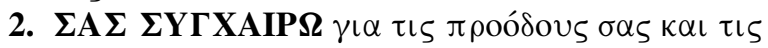

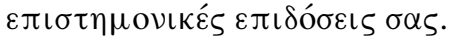

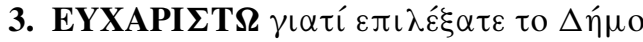

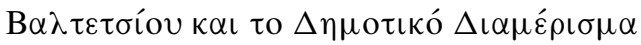

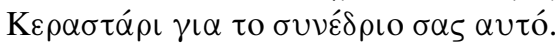

4. EYXOMAI $\varepsilon \pi \iota \tau \cup \chi i ́ \alpha \kappa \alpha \iota \pi \rho o ́ o \delta o$

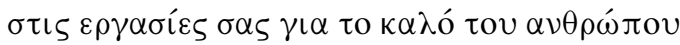

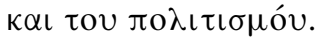

Кú

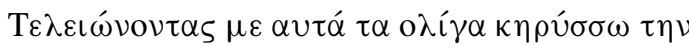

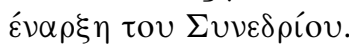

EYXAPI $\Sigma T \Omega$.
Ladies and gentlemen, I shall try to end this speech with these words that show the feelings I have right now:

1. I PAY MY RESPECTS to human and his values which are the highest pitch of Democracy.

2. I CONGRATULATE YOU on your progress and your scientific achievements.

3. THANK YOU for choosing Valtetsi District and the Municipal Compartment of Kerastari for your Convention.

4. I WISH YOU a good luck in your labour for the welfare of the human being and his culture.

Ladies and gentlemen welcome to our land.

With these last words I declare the beginning of your International Convention.

THANK YOU. 